\title{
Interfacial Structures of Polyurethane Thin Films on Various Substrate Materials
}

\author{
Jangsoon KIM, ${ }^{\dagger}{ }^{\dagger \dagger}$ Jaedong CHO, Earle RYBA, and Jianming BAI* \\ Department of Materials Science and Engineering, The Pennsylvania State University, University Park, PA 16802 \\ *High Temperature Materials Laboratory, Oak Ridge National Laboratory, 1 Bethel Valley Road, TN 37831-6062
}

(Received May 13, 2003; Accepted September 16, 2003)

\begin{abstract}
Grazing incidence X-ray diffraction (GIXD) was performed on three polyurethane films, prepared with polyol $\mathrm{OH}$ number of 120,375 , and 600 cast on various substrates, such as silicon, gold, glass, sodium chloride, and zinc phosphated steel. Surface topography did not affect the structural variation of polyurethane films along with the film depth controlled by an angle of incidence. Change of the crystallinity was occurred by a simultaneous change of interplanar spacing, indicating that substrate-induced crystallization took place. The depth that the substrate surface influenced the crystallization of the polyurethane films corresponded to the halfway of the variation of interplanar spacing. The substrate-induced ordering of the polyurethane films varied according to the substrate surface conditions, presumably depending on the attraction force forward the heterogeneities inside the polymer bulk film. It was suggested that the substrate-induced crystallization of the polyurethane on the various substrates depends on the mobility of heterogeneities toward the polar substrate surface rather than other possible mechanisms such as temperature gradient, chemical similarity, lattice match, and thermal shear stress.

KEY WORDS Substrate-Induced Ordering / Polyurethane Thin Film / Grazing Incidence X-ray

Diffraction /
\end{abstract}

When a polymer is used as a facing film on substrate, the mobility of polymer chains is restricted by the presence of the substrate surface. The substrate surface provides the sites where polymer molecules reside. In the case where the polymer chains arrange in a periodic registry, the nucleation and growth of the polymer crystalline phase take place at the substrate surface. It is because the surface has a nucleating efficiency equal to or greater than that of the other nuclei within the polymer film, ${ }^{1}$ the nucleation is heavily favored at the substrate surface, followed by the formation of substrate-induced crystalline region (SICR).

It has been emphasized that the lattice matching is the most important factor for the SICR formation, ${ }^{2-5}$ since the nucleating efficiency increases with closeness of match between the lattice parameters of the substrate and the forming polymer crystal. However, the SICR appears even in little similarity in unit cell parameters, ${ }^{6,7}$ implying that lattice match between the crystallizing polymer and the substrate material is not a necessary condition for the formation of the SICR. Although temperature gradient between the substrate surface and polymer film influences the nucleation and growth process of the SICR, this does not also appear to be necessary for the SICR to form; isothermally crystallized polypropylene has been observed on nylon fiber substrate which has improbable temperature gradient. ${ }^{6}$ Surface energy of the substrate material has been con- sidered as a formation mechanism of the SICR, but both low energy surface (i.e., Terylene) and high energy surface (i.e., graphite) revealed the SICR morphology. ${ }^{7-12}$ Surface topography partially explains the difference in nucleating ability of various substrates, ${ }^{13}$ even though the SICR has been observed even on the surfaces containing no detectable cracks. ${ }^{6,7}$ Shear stress caused by difference in thermal expansion coefficients between the polymer and substrate produces molecular orientation to decrease entropy. Despite the similar thermal expansion coefficients, the SICR has been also found in polypropylene and nylon-66 system. ${ }^{6}$ Mobility of heterogeneities, such as catalyst residues and dust particles within the polymer solution, may adsorb onto the substrate surface. ${ }^{6}$ It presupposes the existence of some type of attractive, possibly polar, forces between the substrate surface and the heterogeneities. The mobility of the heterogeneities decreases at lower crystallization temperature. Overall, the mechanisms for the substrateinduced ordering of polymer molecules tend to be dependent upon the substrate surface and sample preparation condition.

Rigid polyurethanes crystallized by heterogeneous nucleation on the Al surface, resulting in spherulitic interphase. ${ }^{14}$ In order to examine how the polyurethanes crystallize at other substrate materials, this study investigated the structural variation of the polyurethane films coated onto various substrates. The effects of the

\footnotetext{
${ }^{\dagger}$ To whom correspondence should be addressed (Tel: 82-42-866-2528, Fax: 82-42-862-6069, E-mail: jangskim@1gchem.com).

${ }^{\dagger}$ Present Address: Corporate R\&D, LG Chem, Ltd., Research Park, South Korea.
} 
crystalline and amorphous surfaces, the surface topography, and the presence of native surface oxide on the substrate-induced ordering of three polyurethanes were found using depth-controlled grazing incidence X-ray diffraction (GIXD) technique with the substrate materials of silicon, glass, gold, sodium chloride, and zinc phosphated steel. The relation of substrate surface condition to the crystallization of the polyurethane films was determined in terms of variation of crystallinity and interplanar spacing as a function of penetration depth of $\mathrm{X}$-ray. Subsequently, the most probable mechanism for the molecular ordering of these polyurethanes under the various substrate surface conditions was suggested.

\section{EXPERIMENTAL}

\section{Sample Preparation}

Si wafer, Au film sputtered on Si wafer, glass (Corning Glass Works, NY), $\mathrm{NaCl}$ (Harrick Scientific Corporation, NY), and $\mathrm{Zn}$-phosphated steel were used as substrate materials. The $\mathrm{NaCl}$ substrate was cleaved with a sharp knife to obtain a very smooth surface. The Zn-phosphated steel was provided by Air Products and Chemicals Inc. These substrates were cut by appropriate size of $1 \times 1 \mathrm{~cm}$ plate for X-ray measurement. The substrates were cleaned using acetone and ethanol prior to the cast of polyurethane solutions. The polyether polyols used in this study were provided by Arch Chemicals, Inc. The polyol $\mathrm{OH}$ numbers were 120,375 , and 600 , corresponding to polyol molecular weights of 1400,596 , and 374 , respectively. In order to form the urethane linkages, these polyols were reacted with diphenylmethane- $4,4^{\prime}$-diisocyanate (MDI) (Aldrich) in dimethylformamide (DMF) (Aldrich). The $3 \mathrm{wt} \%$ solutions of three polyurethanes, PU-120, PU375 , and PU-600 (named by the polyol $\mathrm{OH}$ number) with an isocyanate index of 105 , were kept at $50^{\circ} \mathrm{C}$ for $1 \mathrm{~min}$ with stirring, followed by casting on the substrates at $50{ }^{\circ} \mathrm{C}$. For these samples, solvent evaporation was performed in air for $72 \mathrm{~h}$ at room temperature and subsequently in vacuum for one week.

\section{X-Ray Scattering}

X-Ray measurements were made with beamline X14A at National Synchrotron Light Source (NSLS, Brookhaven National Laboratory) in Huber Four Circle diffractometer operating in a $\theta / 2 \theta$ geometry. An X-ray energy of $8.048 \mathrm{keV}(\lambda=1.54076 \AA)$ was used. GIXD measurements were carried out using a symmetric fourcircle diffraction geometry in which angles of the incident and diffracted beams with respect to the substrate surface are equal. Seven to ten incident angles were used to examine the variation of the polyurethane crys-
Table I. Critical angles for polyurethane films

\begin{tabular}{cc}
\hline film & critical angle (degree) \\
\hline PU-120 & 0.186 \\
PU-375 & 0.190 \\
PU-600 & 0.203 \\
\hline
\end{tabular}

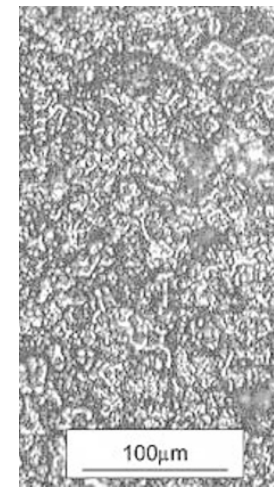

(a)

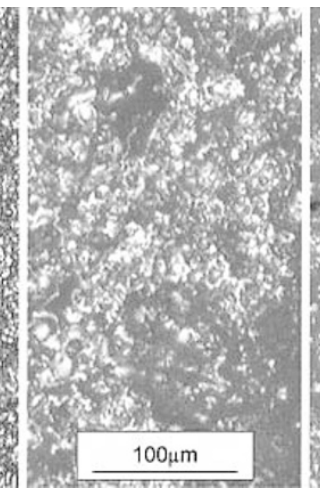

(b)

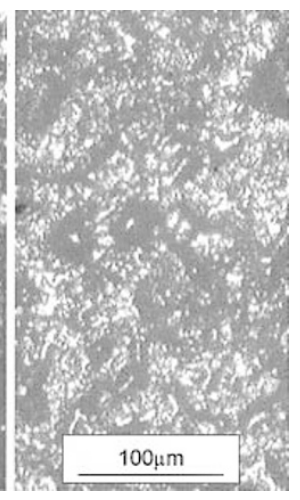

(c)
Figure 1. Observation of PU-120 (a), PU-375 (b), and PU600 (c) film surfaces coated onto $\mathrm{NaCl}$ substrate as representative micrographs to show morphologies of the polymer film surfaces bonded to various substrates.

tal structure along the film depth. The temperature was ambient, and the scan range was fixed between 5 to $45^{\circ}$. Detailed GIXD experimental set-up and methods are shown in ref 15. In order to determine the penetration depth of X-ray, the critical angles (Table I) were measured by specular scans in the $2 \theta$ range of $0.1-2^{\circ}$ for the polyurethane films used in this work. Further, the polymer film thickness was not exactly measured from somewhat irregular surface morphology (Figure 1), but GIXD scans give the approximate thickness with the appearance of reflections from the substrate materials. On the other hand, the polymer surface roughness is significantly important in GIXD scan, because extent of the roughness may determine the scattering profile on the polymer surface. The depth controlled GIXD scan performed in this study is, however, less sensitive to the polymer surface roughness. Instead, the structural variation of polymer film with depth is more important. Therefore, the irregular polyurethane surface observed in Figure 1 does not appreciably affect the following analysis based on the GIXD scan data.

The peaks from the GIXD scans, after background subtraction, were indexed using the diffraction data for a polyurethane-urea given by Ishihara et al. ${ }^{16}$ for monoclinic unit cell with dimensions $a=4.72 \AA, b=11.33 \AA$, $c$ (chain axis) $=11.64 \AA$, and $\gamma=116.5^{\circ}$. The hard segment of this polymer consists of only MDI and terminated urethane-urea functional groups. FT-IR spectra (conducted on a BioRad FTS spectrometer) of all three polyurethane films showed the existence of urea groups which may have been formed from the reaction 
of MDI with water molecules in the air. Thus, the use of the powder pattern of the polyurethane-urea mentioned previously is appropriate.

The GIXD scattering data in the $2 \theta$ scan range of 10 $30^{\circ}$ were curve-fitted along with the best fit in terms of multiple Gaussian peaks, since all the intense crystalline peaks were found in this range. Crystalline peaks were separated from the amorphous halo by the fitting of X-ray scattering results through a program with Gaussian function. ${ }^{17}$ The crystallinity for each sample was measured from the ratio of the area under the crystalline peaks to the total area under the diffraction curve. The interplanar spacing, $d$, was also obtained from the fitted results. The crystallinity and $d$ were plotted as a function of penetration depth of X-ray. The (020) and (021) reflections in the wide angle Xray diffraction showed corresponding intensities with the patterns observed by Ishihara et al., ${ }^{16}$ while these reflections in the GIXD scattering data were not clear to obtain the exact Bragg angle position. Therefore, the fitting result of (020) and (021) reflections from the GIXD scans was used only for the crystallinity calculation.

\section{RESULTS AND DISCUSSION}

GIXD scans determine whether Bragg peaks appear from the bulk polyurethane or all of the polymer plus the substrate by changing the angle of incidence appropriately. The GIXD scan was performed to find the effect of substrate surface on the molecular ordering of polyurethane films coated onto various substrates. Figure 2 exhibits how Si surface affected the molecular ordering of three polyurethanes. PU-375 and PU-600 films show discernible (100) and (021) reflections with the angle of incidence. PU-120 film exhibits the effect of an evident Si surface, in such a way that any peak from the polymer crystalline phases does not appear even at angle of incidence of $0.122^{\circ}$ (below critical angle of PU-120 film) corresponding to scattering from polymer surface. Appearance of a clear (100) reflection begins with X-ray penetration, controlled by angle of incidence. It indicates that the PU-120 film did not crystallize at air facing polymer, and, instead, the substrate-induced ordering was caused by the presence of the Si surface. The PU-375 and PU-600 films crystallized through the entire film with a gradient of crystallinity and variation of interplanar spacing to be discussed in the later part. Although the PU-375 and PU-600 films did not exhibit a remarkable substrateinduced ordering in the GIXD scattering pattern, a clear appearance of (100) and (021) reflections with an angle of incidence is the indication of the Si surface effect.
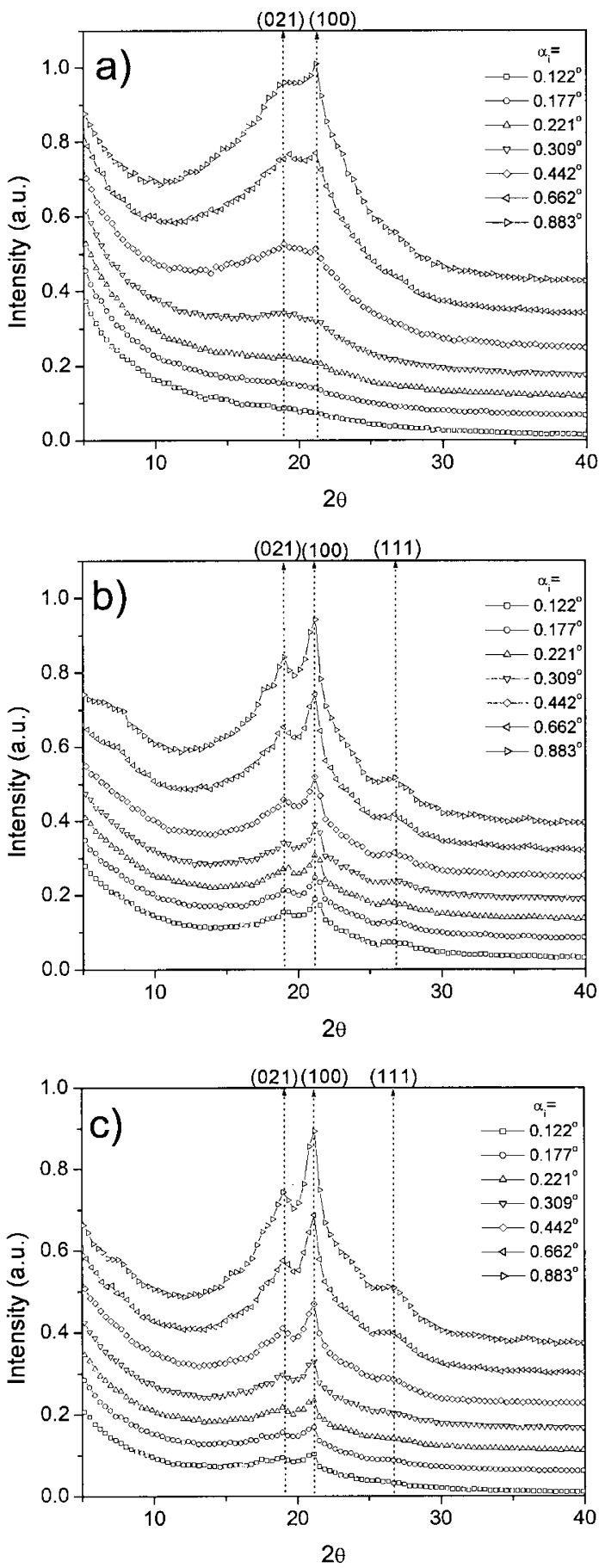

Figure 2. Grazing incidence X-ray scans of the PU-120 (a), PU-375 (b), and PU-600 (c) films coated onto Si substrate at various angles of incidence. The dotted vertical lines indicate polyurethane crystalline peak position.

Glass surface differently affected the crystallization of the polyurethane films in Figure 3. The PU-375 film did not exhibit the crystallization induced by glass substrate, resulting from the similar GIXD scan data at all the angles of incidence. For the PU-120 and PU600 films, the polymer crystalline peaks are discernible with increase of the angle of incidence. The crystallization of the PU-120 and PU-600 films was affected by the presence of glass surface, whereas the reflec- 

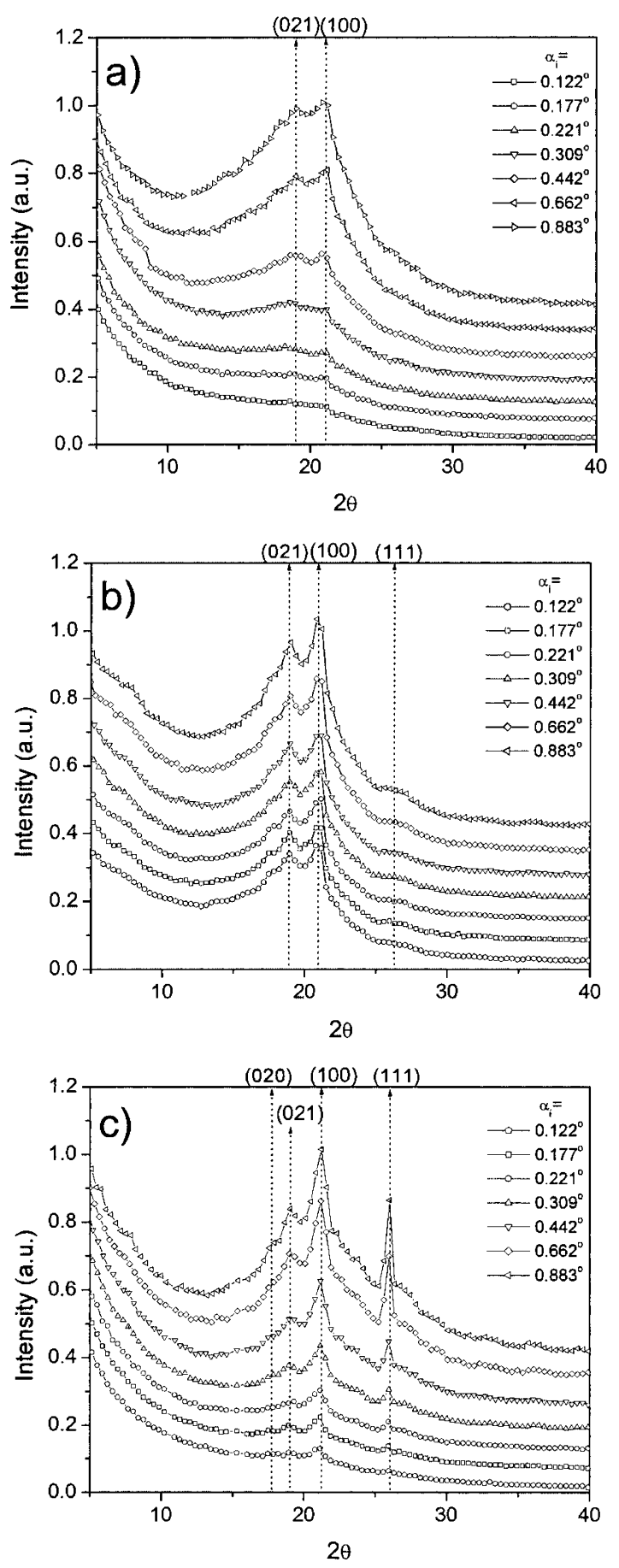

Figure 3. Grazing incidence X-ray scans for the PU-120 (a), PU-375 (b), and PU-600 (c) films coated onto glass substrate at various angles of incidence. The dotted vertical lines indicate polyurethane crystalline peak position.

tions from the PU-375 film appeared by the nucleation and growth of the polymer crystallites at the surfaces of the heterogeneities within the bulk polymer as well as at the glass surface. Existence of (111) peak in the PU-600 film was obvious with the penetration of X-ray radiation, controlled by angle of incidence. It is presumed that additional crystallization and/or interaction between polymer and surface occurred near the interfacial area. This represents an obvious substrate effect
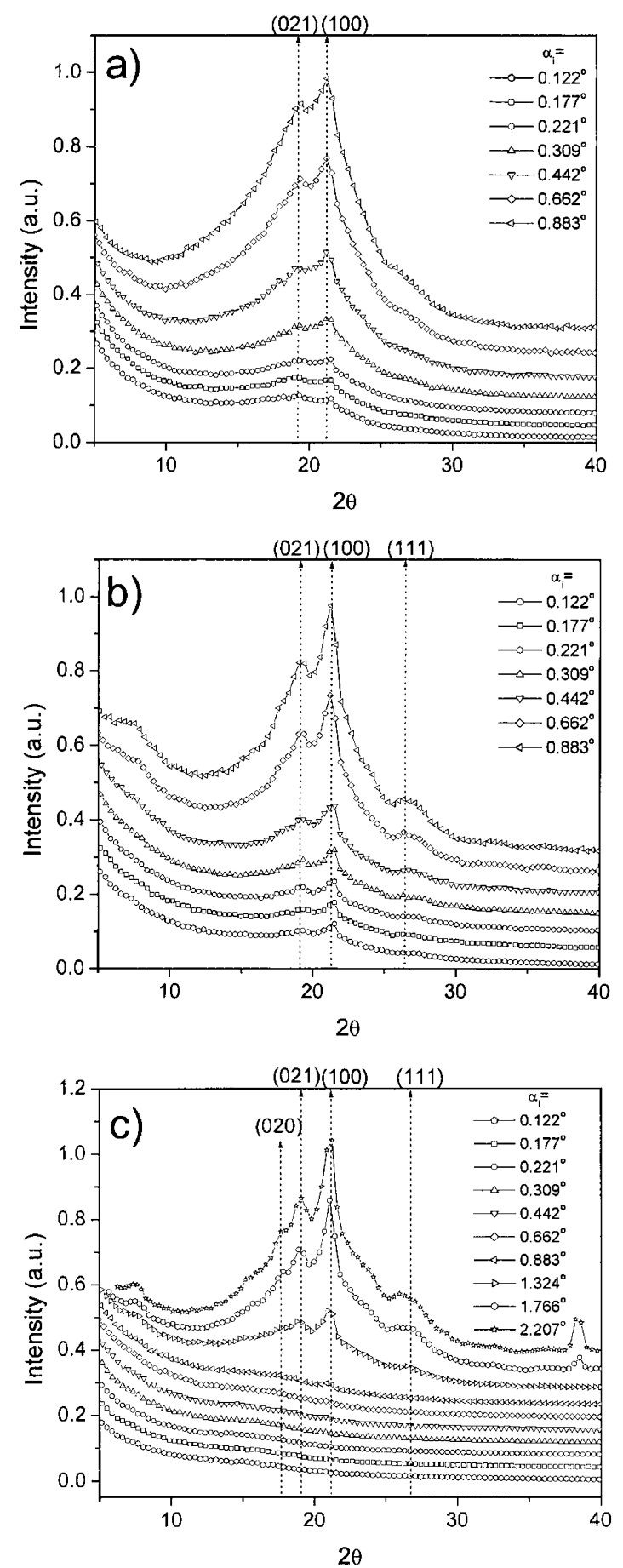

Figure 4. Grazing incidence X-ray scans for the PU-120 (a), PU-375 (b), and PU-600 (c) films coated onto gold substrate at various angles of incidence. The dotted vertical lines indicate polyurethane crystalline peak position.

on the crystallization of the PU-120 and PU-600 films. However, the fact that the SICR effect was not present in the PU-375 film is not clearly understood. This may be related to the difference of the attractive force of the glass surface toward these polymers.

From the GIXD scattering data in Figure 4, all of the three polyurethane films show the Au substrate effect. The PU-600 film exhibits a clear formation of the SICR, in that reflections from the polymer crystalline 
phases appeared only near the Au substrate surface. It indicates that the hard segments of the PU-600 film significantly segregated near the Au surface. The phase separation is probably due to the large attractive force of the Au surface toward the PU-600 film. The Au has non-oxide surface, and its surface is very stable in the case where foreign particles such as dust are removed during the surface cleaning. Therefore, the suggestion that the energy-state of Au substrate surface causes the substrate-induced crystallization does not tend to be appropriate in the polyurethane/Au bond systems.

In order to understand the effect of surface topography on the substrate-induced ordering of polyurethane films, smooth $\mathrm{NaCl}$ and rough $\mathrm{Zn}$-phosphated steel were chosen as the substrate materials. The fractured $\mathrm{NaCl}$ surface, prepared by cleaving with a very sharp razor blade, is very smooth with the roughness around few hundred nanometers, while the treatment of the steel substrate in a zinc phosphate solution provides a very irregular surface with the surface roughness ranges several hundred micrometers. The crystallization of the PU-120 film was not influenced by the presence of the $\mathrm{NaCl}$ surface as shown in Figure 5. The $\mathrm{NaCl}$ surface did not provide the heterogeneous nucleation sites for the molecular ordering of the PU-120 film. The crystallization of the PU-120 film was completely dependent upon the existence of unknown heterogeneities within the bulk film. The GIXD scattering for the PU-375 film represents a typical crystallization phenomenon of polymer film; the crystallization inside the polymer bulk film as well as at the $\mathrm{NaCl}$ surface occurred simultaneously. The $\mathrm{NaCl}$ surface led to a distinct substrateinduced ordering of the PU-600 film. Likewise the GIXD scans for the PU-600 film at the glass surface, a clear (111) reflection and another reflection around $2 \theta=15^{\circ}$ showed up with the penetration of X-ray radiation, controlled by angle of incidence. One wonders how the polymer chains align on the very smooth cleaved $\mathrm{NaCl}$ surface. Some part of the $\mathrm{NaCl}$ surface was, presumably, dissolved into the solvent dimethylformamide (DMF), resulting in the irregular surface. Hence, the substrate-induced ordering of the PU-600 film originates likely from the contribution of the surface irregularity such as ledges and crevices. The dissolved $\mathrm{NaCl}$ particles are also possible heterogeneities to form this SICR. The steel surface, treated using a zinc phosphate solution, consists of a lot of pits, cracks, and other defects. ${ }^{18}$ This rough steel surface caused the structural variation of all of the polyurethane films (see Figure 6). The rough surface could provide a lot of nucleation sites where the polyurethane hard segments sit to arrange into the periodic registry. In particular, the influence of surface roughness was remarkable in the
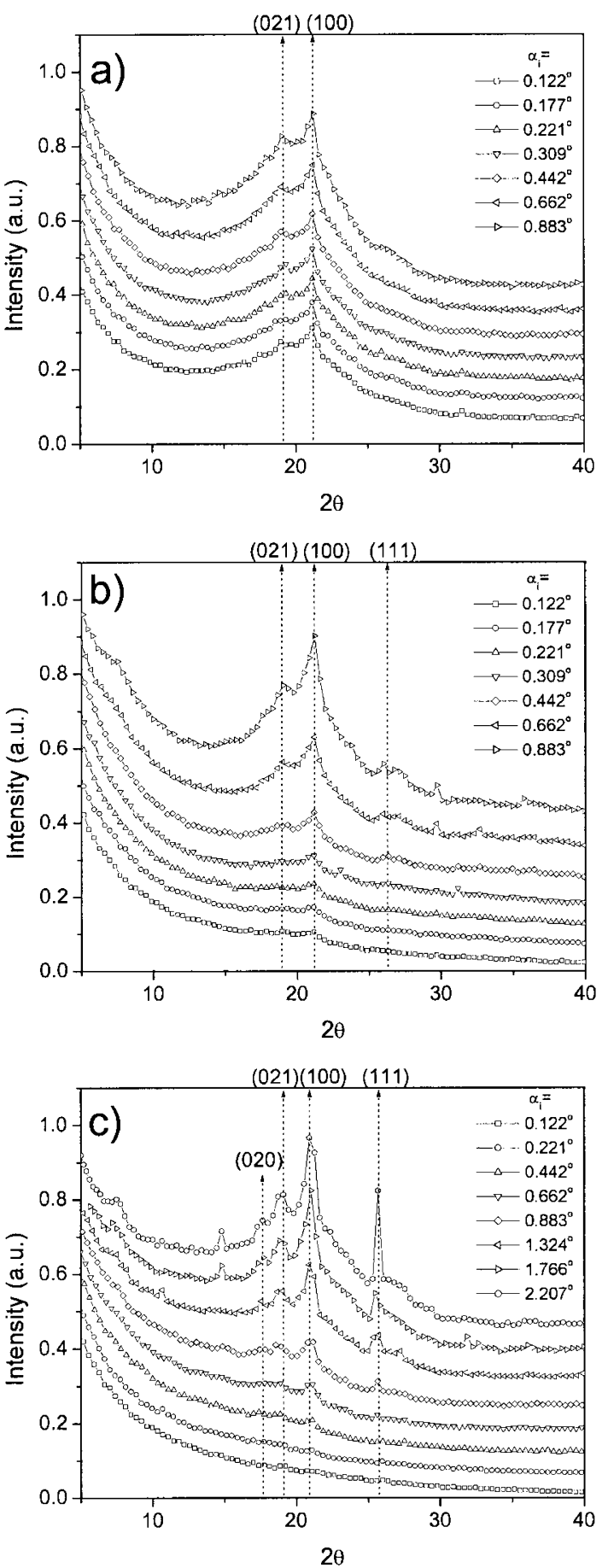

Figure 5. Grazing incidence X-ray scans for the PU-120 (a), PU-375 (b), and PU-600 (c) films coated onto $\mathrm{NaCl}$ substrate at various angles of incidence. The dotted vertical lines indicate polyurethane crystalline peak position.

PU-600 film. The PU-600 film exhibited a sudden appearance of (100), (021), and (111) reflections at the angle of incidence of $0.309^{\circ}$. The presence of the $\mathrm{Zn}$ phosphated steel surface persisted up to the penetration depth of $3.563 \mu \mathrm{m}$ corresponding to this angle of incidence. The intensities of (021) and (111) reflections increased in all of the polyurethane films with X-ray radiation depth. It implies that the presence of the $\mathrm{Zn}$ phosphated steel surface affected the arrangement of 

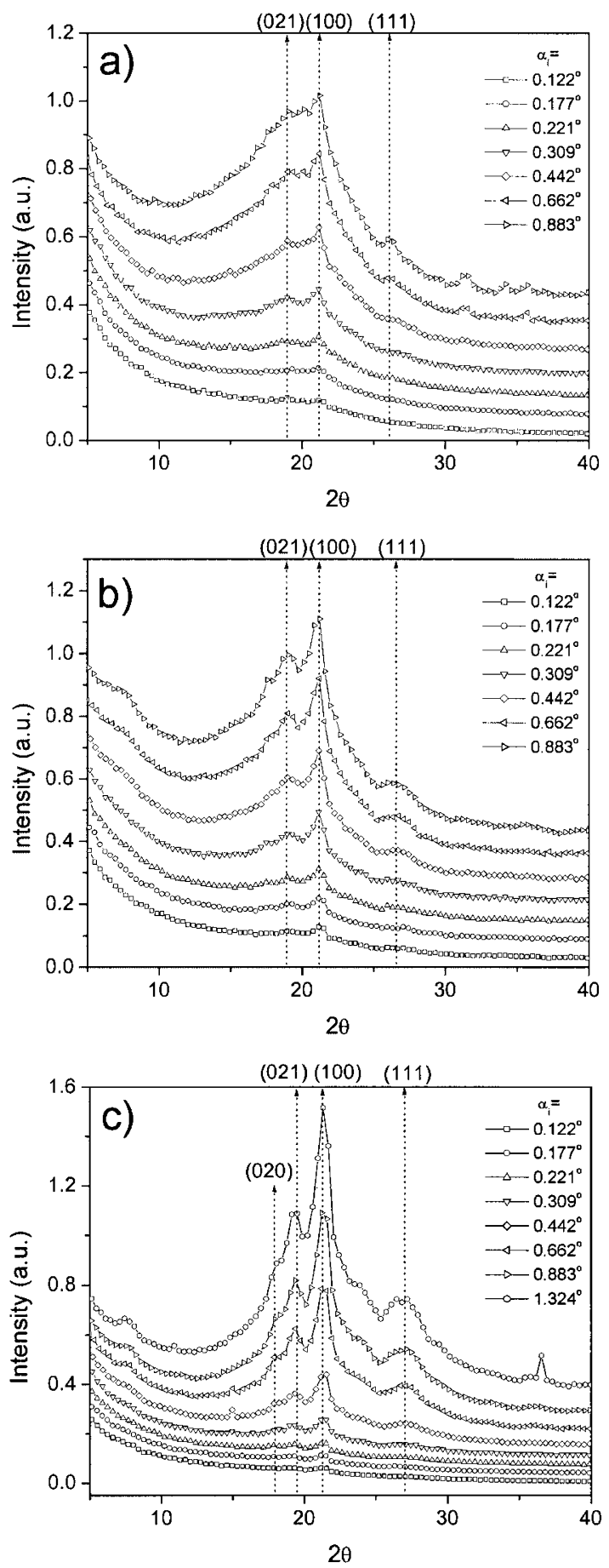

Figure 6. Grazing incidence X-ray scans for the PU-120 (a), PU-375 (b), and PU-600 (c) films coated onto Zn-phosphated steel substrate at various angles of incidence. The dotted vertical lines indicate polyurethane crystalline peak position.

polymer chains, and, thus, further nucleation of polymer crystalline phase occurred near the steel surface. However, the polyurethane films/Zn-phosphated steel bonds did not exhibit any specific crystallographic feature in comparison with the polyurethane films $/ \mathrm{NaCl}$ bonds, even though it is expected that variety of nucleation sites at the Zn-phosphated steel surface may be able to lead to other molecular orders. Therefore, the surface geometry is not an appropriate parame-
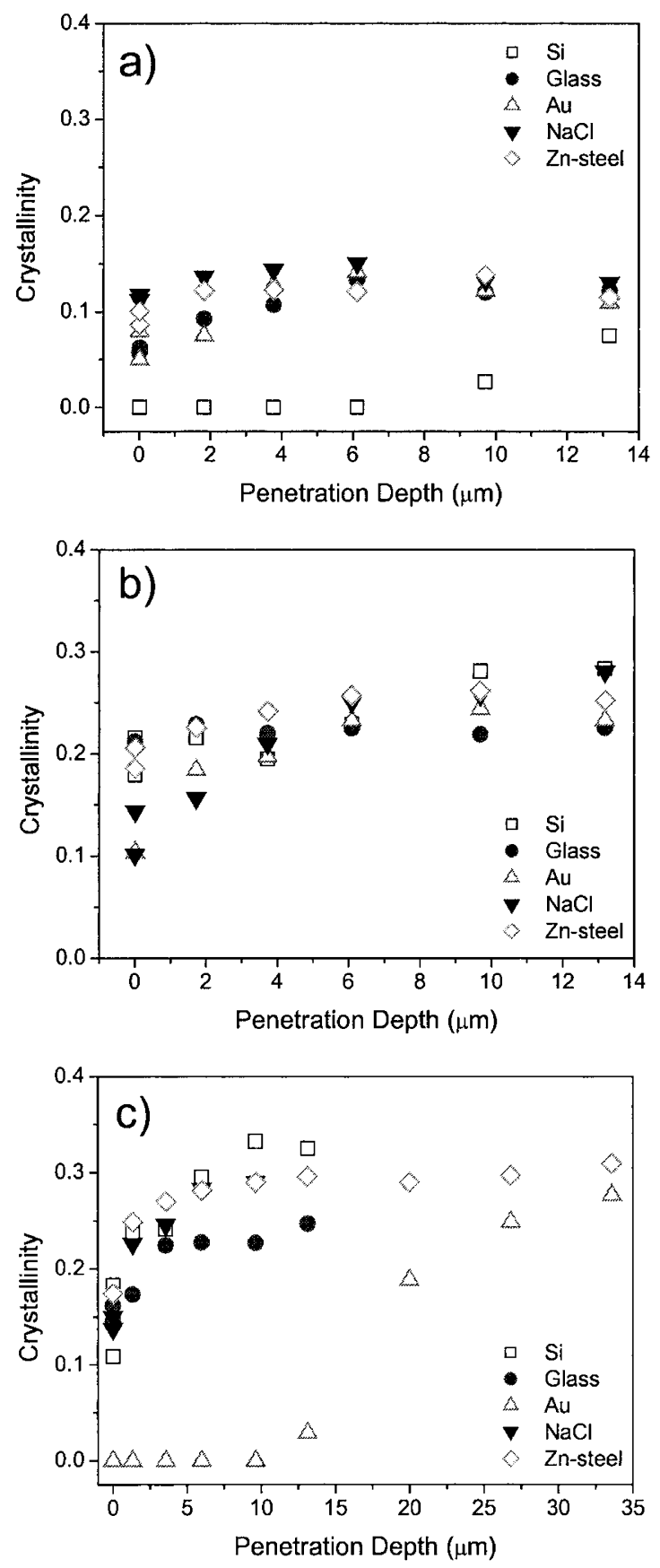

Figure 7. Variation of crystallinity along depth of (a) PU-120, (b) PU-375, and (c) PU-600 films from air facing polymer surface to polymer/substrate interface.

ter to cause the substrate-induced crystallization of the polyurethane films.

Figure 7 exhibits the change of the crystallinity for the polyurethane films, coated onto the various substrates, as a function of penetration depth of X-ray. When incident angle $\alpha_{i}$ were known, the penetration depth of X-ray can be calculated by

$$
\Lambda=\left(\frac{\lambda}{\sqrt{2} \pi}\right)\left\{\left[\left(\alpha_{i}^{2}-\alpha_{c}^{2}\right)^{2}+(2 \beta)^{2}\right]^{1 / 2}-\left(\alpha_{i}^{2}-\alpha_{c}{ }^{2}\right)\right\}^{-1 / 2}
$$

where the extinction coefficient arises due to the ad- 
sorption of X-rays by material. The critical angle $\alpha_{c}$ was measured by specular scan in Table I. As shown in the GIXD scan results, the crystallinities of the PU-120 film at the Si surface and the PU-600 film at Au surface are zero near polymer surface, followed by the sudden increase with approaching substrate surface. This is a clear evidence of the effect of the substrate surface on the formation of the SICR. It is thought that a significant segregation of the hard segments took place near the polymer/substrate interface, even though the reason is not understood. The PU-120 film crystallized in a similar crystallinity of below $15 \%$ degree on all of the substrate materials. The $\mathrm{NaCl}$ and $\mathrm{Zn}$-phosphated steel surfaces negligibly affected the crystallization of the PU-120 film, resulting in the similar crystallinity through a whole film. It implies that the extent of molecular ordering of the PU-120 film was not related to the surface topography. This variation of crystallinity along with the film depth correlates with the variation of $d_{(100)}$ shown in Figure 8 such that the samples with a constant crystallinity through entire film exhibits a simultaneous constant $d_{(100)}$. Factor et al. ${ }^{18}$ suggested that "the $d$ that is halfway between the bulk and surface values provides a rough estimate for the depth to which the surface effects persist'. In fact, polymer molecules near the substrate surface are subject to be strained, since the substrate surface restricts the motion of the polymer chains. This causes different molecular orders from the stress free bulk order, and results in the shift of interplanar spacing $d$. These suggest that large variation of $d$ means the presence of the great substrate effect on the polymer molecular ordering, while constant $d$ represents the absence of influence of the substrate surface on the polymer crystallization. From the invariance of $d_{(100)}$ for the PU-120 films at $\mathrm{NaCl}$ and $\mathrm{Zn}$-phosphated steel surfaces, two substrates did not take part in the polymer molecular ordering. In other words, the molecular ordering of the PU-120 film was not influenced by surface topography. This was confirmed by similar GIXD scattering data through the entire film, as shown in Figures 5 and 6. The substrate-induced ordering of the PU-120 film greatly occurred when the Si substrate was used, presumably, resulting from the existence of the native silicon oxide. The natural oxide may have caused the significant segregation of the polyurethane hard segments. Since the amorphous glass and the $\mathrm{Au}$ do not form such an oxide surface, these substrates do affect molecular ordering of the PU-120 film in less degree.

Overall, the PU-375 films on all of the substrates crystallized in a similar range of about $30 \%$, regardless of the surface condition. However, the crystallinity through a whole film varies with the substrate materials.
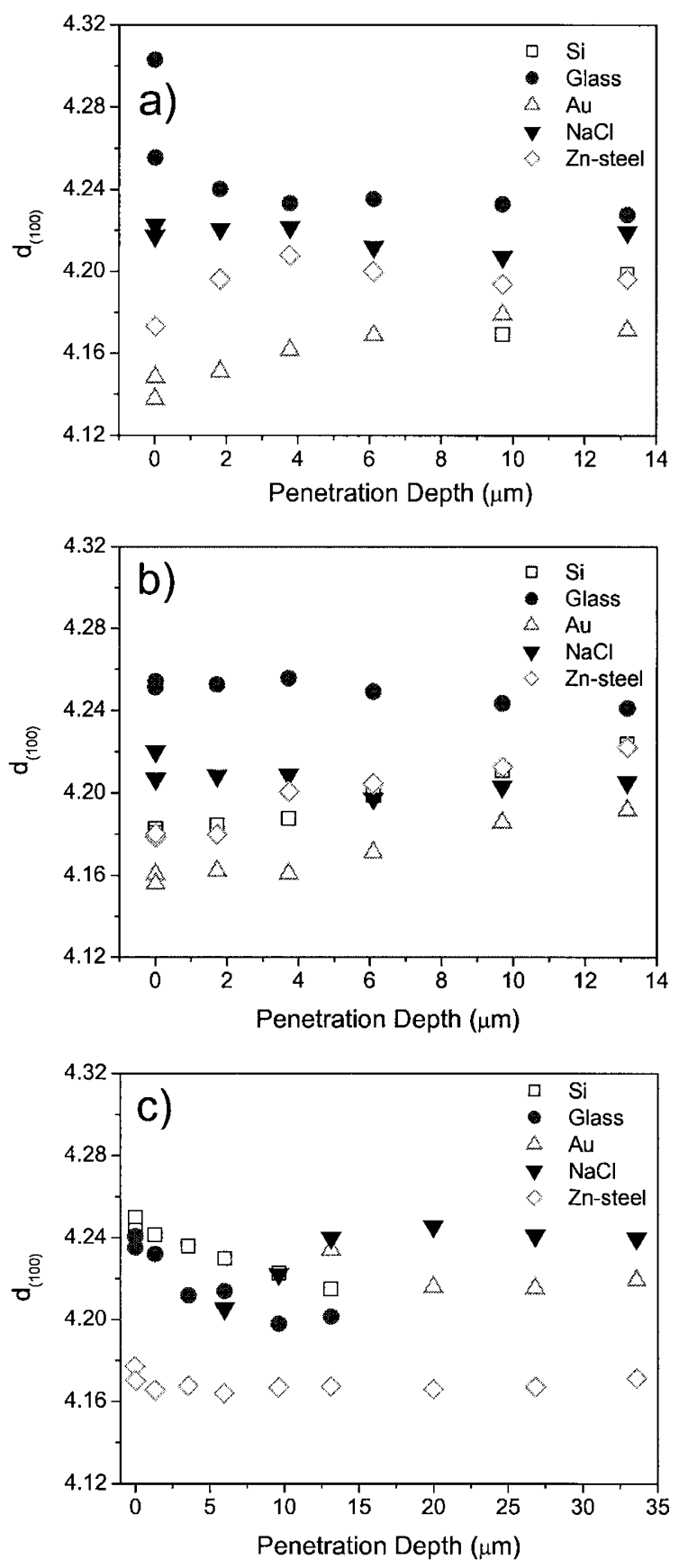

Figure 8. Variation of interplanar spacing $d$ for reflection (100) as a function of penetration depth, controlled by angle of incidence, in (a) PU-120, (b) PU-375, and (c) PU-600 films.

The PU-375 film on glass surface exhibits a constant crystallinity from air facing polymer surface down to polymer/substrate interface. It indicates that the amorphous surface did not lead to the substrate-induced ordering of the PU-375 film. The crystallization process depends only on the existence of heterogeneities within the polymer bulk film. This is very similar to the crystallization of the PU-120 films at the $\mathrm{NaCl}$ and $\mathrm{Zn}-$ phosphated steel surfaces. The constant crystallinity through a whole film takes place with the simultaneous invariance of $d_{(100)}$. Other substrate materials affected the molecular ordering of the PU-375 film, in that the 
crystallinity increases with the penetration depth of Xray. The amorphous surface did not attract the chains of the PU-375 polymer. The surface topography and presence of surface oxide do not affect the formation of SICR. It is noted that the $\mathrm{Au}$ and $\mathrm{NaCl}$ exhibited the great substrate effect on the crystallization of the PU375 film.

The effect of the substrate material on the crystallization of the PU-600 film was evident. In particular, the formation of the SICR of the PU-600 film induced by the non-oxide Au surface was distinct, in that the crystallinity was close to zero from air facing polymer surface down to a certain film depth. The Au substrate that does not form a natural surface oxide provided for better sites for the segregated hard segments of the polyurethane to nucleate and grow. On the other hand, the extent of molecular ordering of the PU-600 film at the smooth $\mathrm{NaCl}$ surface was not quite different from that of the rough $\mathrm{Zn}$-phosphated steel surface. The surface topology is, apparently, not an important factor to dominate the crystallization process of the PU-600 film. The surface takes part in only providing the sites where the polymer chains get into the periodic registry. The crystallization of the PU-120 and PU-375 films was dependent upon the condition of substrate materials, whereas the PU-600 film crystallized regardless of the substrate surface condition. The PU- 375 film on an amorphous glass and the PU-120 film on a $\mathrm{NaCl}$ exhibited invariance of $d_{(100)}$, showing constant crystallinity with the penetration depth, controlled by angle of incidence. It indicates that there was no substrate effect on the polyurethane crystallization. Except for both samples, other samples exhibited the initial increase or decrease of $d_{(100)}$, followed by constant $d_{(100)}$. Providing that the suggestion from Factor et al. ${ }^{19}$ is correct, the substrate surfaces affect the crystallization of the polyurethane up to a significant thickness away from the polymer/substrate interface.

The results from the effect of surface condition on the crystallization of polyurethane films were compared with the formation mechanisms of the substrateinduced crystallization. Temperature gradient between the polyurethane films and the substrate surfaces was not present in the sample preparation, because the preheating temperature of the substrates was the same as that of the polymer solution prior to casting. Thermal shear stress is also not appropriate for evolution of the substrate-induced crystallization of the polyurethane films. These terms should be considered in the case where polymer melt under exothermic reaction during polymerization is coated onto the substrate. Lattice match is neglected in that lattice parameters of the polyurethane crystallites and the substrates materials used in this study are not similar. Wang et al. ${ }^{20}$ reports that surface topography dominates the nucleation process of the polymer crystallization, since small ridges, pits, cracks, and valleys are usually present on most of substrate surfaces. However, the present study reveals that the polyurethane films crystallized in a similar range against both rough $\mathrm{Zn}$-phosphated steel and smooth $\mathrm{NaCl}$ substrates. The distribution of the crystallographic orientation of the hard segments is not quite different. Obviously, the surface topography did not affect the substrate-induced ordering of the polyurethane molecules. Even though the $\mathrm{NaCl}$ surface is not perfectly clean without defects, the surface roughness is quite different between $\mathrm{NaCl}$ and $\mathrm{Zn}$-phosphated steel. The SICR formation of the polyurethane films could not be explained in terms of the surface geometry. The polyurethane and the substrate materials used in this study are chemically quite different. Nevertheless, the polyurethane films still crystallized near substrate surface according to the formulation level and substrate materials. Therefore, the chemical similarity as the formation mechanism of the SICR of the polyurethanes is not appropriate for the materials system in this study. The mobility of heterogeneities is a presumable factor that leads to the substrate-induced ordering of the polyurethane molecules. The set of samples was prepared with the same dish and polymer solution; there is no difference of the polymer properties between each samples. The polar force of the substrate to attract the heterogeneities within the bulk polymer toward the substrate surface might be different, even though the polar ability of those surfaces is not known.

\section{CONCLUSIONS}

In order to understand the formation mechanism of the substrate-induced crystallization of polyurethane films at various substrates, a grazing incidence X-ray diffraction was performed. Depending on the formulation level, the polyurethane films exhibited different extent of the substrate-induced molecular ordering. Interestingly, polymer films that did not represent the substrate effect exhibited constant crystallinity and simultaneous invariance of $d_{(100)}$ through entire film. Providing that the variation of interplanar spacing is indirect proof of the substrate effect, the constant crystallinity and similar GIXD scan data along with the film depth support the absence of influence of the substrate surface. It was found that surface topography did not dominate structural variation of the polyurethanes, resulting from the GIXD scan data for polyurethane films at $\mathrm{NaCl}$ and $\mathrm{Zn}$-phosphated steel surfaces. The existence of surface oxide tends to be a considerable pa- 
rameter, capable of developing the substrate-induced molecular ordering. Mobility of heterogeneities within raw chemicals during polymerization toward the substrate surface is, apparently, a crucial factor to cause the surface-induced ordering. Although the attractive force of each substrate materials for the heterogeneities is not clearly known, the mobility may be determined by the surface polarity. Consequently, formulation level and sample preparation dominate the crystallization of the polyurethane films on the solid substrate.

Acknowledgments. This work was supported by Air Products and Chemicals Inc. This research was performed in part on beamline X14A of the National Synchrotron Light Source, Upton, NY. X14A is operated by the High Temperature Materials Laboratory User Program of Oak Ridge National Laboratory which is sponsored by the Assistant Secretary for Energy Efficiency and Renewable Energy, office of Transportation Technology under contract No. DE-AC0500OR22725. The NSLS synchrotron facility is sponsored by the Office of Science, US Department of Energy under grant no. DE-AC02-76CH00016.

\section{REFERENCES}

1. J. R. Shaner and R.D. Corneliussen, J. Polym. Sci., Polym. Lett. Ed., 1611 (1972).

2. H. J. Kestenbach, J. Loos, and J. Petermann, Polym. Eng. Sci., 38(3), 478 (1998).
3. S. E. Rickert and E. Baer, J. Appl. Phys., 47(10), 4304 (1976).

4. T. Takahashi, M. Inamura, and I. Tsujimoto, J. Polym. Sci., Polym. Lett. Ed., 8, 651 (1970).

5. F. Tuinstra and E. Baer, J. Polym. Sci., Polym. Lett. Ed., 8, 861 (1970).

6. D. Campbell and M. M. Qayyum, J. Polym. Sci., Polym. Phys. Ed., 18, 83 (1980).

7. A. M. Chatterjee, F. P. Price, and S. Newman, J. Polym Sci., Polym. Phys. Ed., 13, 2369 (1975).

8. D. G. Gray, J. Polym. Sci., Polym. Lett. Ed., 12, 645 (1974).

9. D. R. Fitchmun and S. Newman, J. Polym. Sci., A-2, 8, 1545 (1970).

10. H. Schonhorn, J. Polym. Sci., Polym. Lett. Ed., 5, 919 (1967).

11. H. Schonhorn, Macromolecules, 1, 151 (1968).

12. J. P. Luongo and H. Schonhorn, J. Polym. Sci., A-2, 6, 1649 (1968).

13. T. Hata, K. Ohsaka, T. Yamada, K. Nakamae, N. Shibata, and T. Matsumoto, in “Adhesion International 1993", L. H. Sharpe Ed., Gordon and Breach Pub., Amsterdam, 1996, pp. 125135.

14. J. Kim and E. Ryba, J. Adhes. Sci. Technol., 15(14), 1747 (2001).

15. J. Kim, E. Ryba, and J. Bai, Polymer, 44, 6663 (2003).

16. H. Ishihara, I. Kimura, and N. Yoshihara, J. Macromol. Sci., Phys., B22, 713 (1983-1984).

17. Microcal Origin, version 6.00, Microcal Software, Northhampton, MA (1999).

18. J. Kim, E. Ryba, J. Bai, and J. Miller, J. Adhes. Sci. Technol., 17(14), 1351 (2003).

19. B. J. Factor, T .P. Russell, and M. F. Toney, Macromolecules, 26, 2847 (1993).

20. C. Wang and C. R. Liu, Polymer, 40, 289 (1999). 